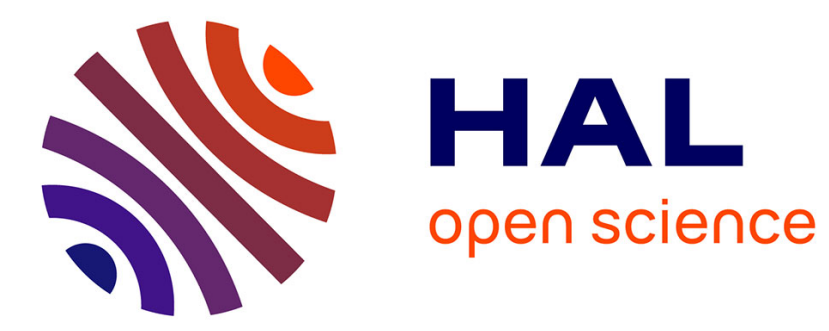

\title{
Comparative study of methods for optimization of electromagnetic devices with uncertainty
}

\author{
Siyang Deng, Stephane Brisset, Stephane Clenet
}

\section{To cite this version:}

Siyang Deng, Stephane Brisset, Stephane Clenet. Comparative study of methods for optimization of electromagnetic devices with uncertainty. COMPEL: The International Journal for Computation and Mathematics in Electrical and Electronic Engineering, 2018, 37 (2), pp.704-717. 10.1108/COMPEL11-2016-0502 . hal-01858676

\section{HAL Id: hal-01858676 https://hal.science/hal-01858676}

Submitted on 21 Aug 2018

HAL is a multi-disciplinary open access archive for the deposit and dissemination of scientific research documents, whether they are published or not. The documents may come from teaching and research institutions in France or abroad, or from public or private research centers.
L'archive ouverte pluridisciplinaire HAL, est destinée au dépôt et à la diffusion de documents scientifiques de niveau recherche, publiés ou non, émanant des établissements d'enseignement et de recherche français ou étrangers, des laboratoires publics ou privés. 


\title{
COMPARATIVE STUDY OF METHODS FOR OPTIMIZATION OF ELECTROMAGNETIC DEVICES WITH UNCERTAINTY
}

\author{
Siyang DENG, Stéphane BRISSET and Stéphane CLENET \\ Univ. Lille, Centrale Lille, Arts et Metiers ParisTech, HEI, EA 2697 - L2EP - Laboratoire \\ d'Electrotechnique et d'Electronique de Puissance, F-59000 Lille, France \\ E-mail: siyang.deng@centralelille.fr
}

\begin{abstract}
This paper compares different probabilistic optimization methods dealing with uncertainties. Reliability-Based Design Optimization is presented as well as various approaches to calculate the probability of failure. They are compared in terms of precision and number of evaluations on mathematical and electromagnetic design problems to highlight the most effective methods.
\end{abstract}

Keywords: Reliability-based design optimization, uncertainty, reliability, safety transformer.

\section{INTRODUCTION}

In most optimization problems, the variables are usually considered as deterministic, i.e. without any variability. This traditional Deterministic Design Optimization (DDO) addresses only the performances but not the reliability and robustness. Since, the manufacturing process, the characteristics of materials, and the dimensions undergo variability, the device performances are in practice not deterministic but uncertain. Therefore, uncertainties related to design parameters are more and more taken into account in all engineering fields.

Various formulations are available in the literature to express optimization problems with uncertainty, which can be mainly divided into Worst-Case Optimization (WCO), Robust Design Optimization (RDO), Reliability-Based Design Optimization (RBDO) and Reliability-Based Robust Design Optimization (RBRDO). WCO is a non-probabilistic approach that is based on minimax problem formulation. For instance, [1] solves a multi-objective problem that aims to minimize the objective function, its maximum feasible value in a surrounding box, and the greatest component of the objective function derivative in the surrounding box.

The three others are probabilistic approaches that quantify the uncertainty of quantities of interest by probability distribution functions. RDO minimize a weighted sum of the mean value and variance of the objective function subject to deterministic constraints. RBDO minimize the mean value of the objective function subject to constraints on the probability of failure, i.e. constraint violation. Finally, RBRDO [2] integrates both last formulations by changing the objective function and constraints at the same time.

A comparative study [3] of two RBDO Double-Loop Methods (DLM) with Monte Carlo Simulation (MCS) shows the interest of RBDO-DLM compared to MCS that requires a very large sampling to be accurate. However, other RBDO approaches such as Single-Loop (SLM) and Sequential Decoupled Methods (SDM) were not simultaneously investigated and this paper proposes to compare 6 algorithms belonging to the three aforementioned RBDO approaches with MCS in order to highlight the most accurate and the less time consuming. This is performed with a simple mathematical model and the multidisciplinary optimization problem of a safety transformer with uncertainty.

The paper is organized into three parts. Chapter 2 introduces the different categories of RBDO approaches. Two examples are detailed in the chapter 3 and used to compare the different methods. Last chapter is the conclusion.

\section{RELIABILITY-BASED DESIGN OPTIMIZATION}

The original formulation without any uncertainty or DDO is expressed as:

$$
\begin{gathered}
\min _{d} f(d) \\
\text { s.t. } g(d) \leq 0 \\
d^{L} \leq d \leq d^{U}
\end{gathered}
$$

where $d$ is the input design variable, $f(\cdot)$ and $g(\cdot)$ are the objective function and the inequality constraint, and $d^{L}, d^{U}$ represent the lower and upper bounds of $d$, respectively.

As the variability of the design variables is taken into account, the original deterministic input parameter $d$ should be replaced by a random input parameter $X$, which follows the normal law in this paper for simplicity. The mean value of $X$ is denoted $d$ and is the unknown of the new design variables, the standard deviation is 
denoted $\sigma$ and is considered constant which means that the variability of the input parameter $X$ doesn't depend on its mean.

The method RBDO aims to find the optimal design with the allocation of a target reliability level. The probability of failure is close to the number of points in a sampling around the mean value that fall within the reliable domain. This means that RBDO attempt to find the optimal design with the reliability that the probability of failure must be smaller than a given target value. The formulation of RBDO is as follows:

$$
\begin{gathered}
\min _{d} f(d) \\
\text { s.t. } P_{f}(g(x)>0) \leq P_{t} \\
d^{L}+\beta_{t} \cdot \sigma \leq d \leq d^{U}-\beta_{t} \cdot \sigma
\end{gathered}
$$

where $P_{f}$ is the probability of failure, $\beta_{t}$ is the reliability index, and $P_{t}=\Phi\left(-\beta_{t}\right)$ the target value for the probability of failure. The RBDO uses probabilistic constraints to make sure that the design variables satisfy a desired reliability level while minimizing the function objective.

\subsection{Probability of failure}

The probability is the likelihood of an event, estimated by a real number between 0 and 1 . The Probability Density Function (PDF) and the Cumulative Distribution Function (CDF) define the occurrence of stochastic quantities inherently uncertain. The statistical description of a random variable $X$ given by the CDF $F_{\mathrm{x}}$ or $\operatorname{PDF} f_{\mathrm{X}}$ is expressed as follows:

$$
P[X \leq x]=F_{X}(x)=\int_{-\infty}^{x} f_{X}(\tau) d \tau
$$

where $P$ is the probability of occurrence of an event.

In the field of electromagnetic device manufacturing, the system ability to satisfy consumer's demand or operating constraints is important. The reliability means that designers should reduce the probability of failure as much as possible. The determination of the reliability of the system is based on the limit state function. Each constraint $g(X) \leq 0$ can separate the domain of $X$ into three parts: the limit state curve is $g(X)=0$, the domain where $g(X)>0$ is the failure domain, on the contrary the security domain represents the area $g(X)<0$.

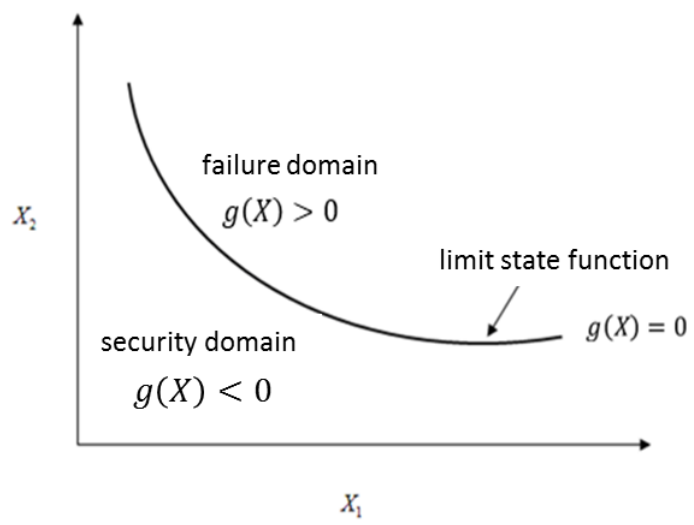

Figure 1. The failure domain, security domain, and limit-state curve.

The probability of failure is the probability of the event $g(X)>0$. It is calculated with the integral:

$$
P_{f}=P[g(X)>0]=P\left[X \in D_{f}\right]=\int_{D_{f}} f_{X}(x) d x
$$

where $D_{f}$ is the failure domain. Because the computational burden is heavy with numerous random parameters, direct integration is almost impossible and thus Monte-Carlo Simulation (MCS) or other techniques such as FirstOrder Reliability Method (FORM) [4], [5] is often used to calculate an approximation of $P_{f}$.

FORM and inverse FORM are based on an isoprobalistic transformation to have a normalized vector of statistically independent random variables $U$ instead of the initial input parameter $X$.

For the Gaussian vector $X$ in this paper, the transformation $T$ is as follows:

$$
T: U=(X-d) / \sigma
$$




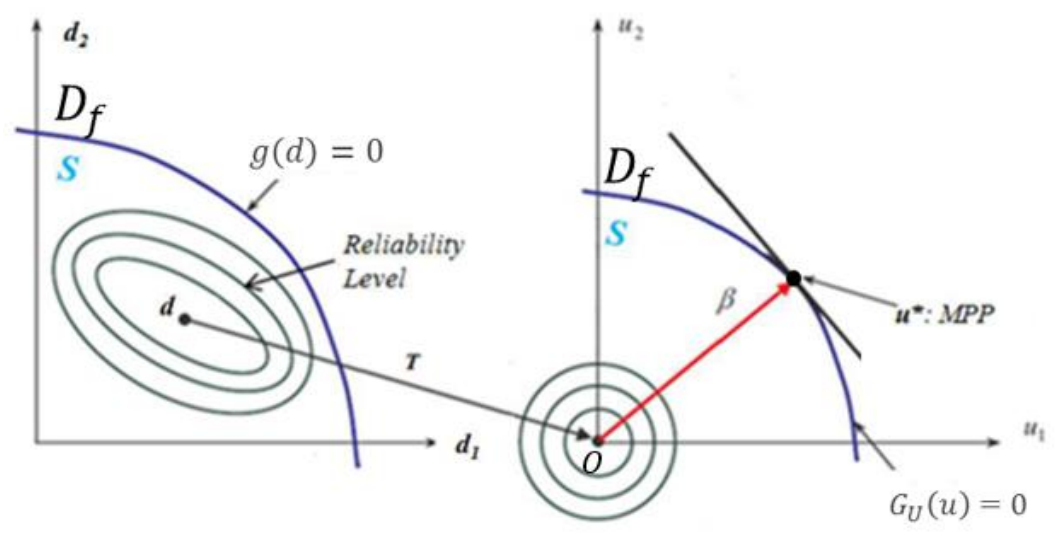

Figure 2. The first order reliability method [17].

Then the limit state function changes from $g(d)=0$ to $G_{U}(u)=0$ and the CDF from $F_{X}$ to $F_{U} . G_{U}(u)$ is defined as the performance function, and the FORM or inverse FORM method uses a linear approximation to replace the real performance function at the Most Probable Point of failure (MPPF) in U-space. The MPPF $u^{*}$ is the one that minimize the distance between the origin $O$ and $G_{U}(u)=0$. After $u^{*}$ is found, the limit-state function is replaced by a tangent hyperplane crossing $u^{*}$. So the probability of failure is calculated by:

$$
P_{f} \approx \int_{\tilde{G}_{U}(u)>0} f_{U}(u) d u=\Phi(-\beta)
$$

where $\tilde{G}_{U}(u)=0$ is the hyperplane that approximates the limit-state function $G_{U}(u)=0, \Phi$ is the standard Gaussian cumulative distribution function, and $\beta=\|u\|$ is the reliability index which is equal the norm of $u$.

The formulation (2) is the basis of all RBDO methods and can be solved by different approaches which are usually separated in three main categories: double-loop, single-loop, and sequential decoupled methods. The following sections introduce the principle of these different categories and present some approaches for each of them.

\subsection{Double-loop methods}

Double-loop methods use two loops to solve RBDO problems: the inner loop aims to analyze reliability of the chosen configuration and to calculate the probability of failure using FORM or inverse FORM; the outer loop seeks the mean values of input designs that minimize the objective function and constrain the probability of failure computed by inner loop.

There are several approaches for double-loop methods, the most popular are Reliability Index Approach (RIA) and Performance Measure Approach (PMA) [3], [6].

\subsubsection{Reliability Index Approach (RIA)}

RIA uses the FORM to calculate the reliability index in the inner loop:

$$
\begin{gathered}
\beta=\min _{u}\|u\| \\
\text { s.t. } G_{U}(u)=0
\end{gathered}
$$

The outer loop of RIA, any constrained non-linear algorithm like SQP or others can be chosen to minimize the objective function $f$ with the index $\beta$ given by the FORM:

$$
\begin{gathered}
\min _{d} f(d) \\
\text { s.t. } \begin{aligned}
g \leq 0 \\
\beta \geq \beta_{t}
\end{aligned} \\
d^{L}+\beta_{t} \cdot \sigma \leq d \leq d^{U}-\beta_{t} \cdot \sigma
\end{gathered}
$$

where $g<0$ is used to restrict the because the definition in equation (6) only validate if the origin is located in the security domain.

\subsubsection{Performance Measure Approach (PMA)}

In former formulation of RBDO, optimization is carried out with the limitation of the reliability index that must be greater than or be equal to a target value. The calculation of this index leads to the search for the MPPF. In contrast, for PMA formulation, optimization is formulated with the limitation of maximum performance with a given value of reliability index. The search of this maximum performance is to maximize the function $G_{U}$ 
with the limitation that reliability index must achieve the target value. This approach is considered as the inverse of the FORM approximation [7], [8].

So the outer loop becomes:

$$
\begin{gathered}
\min _{d} f(d) \\
\text { s.t. } \quad G_{p}\left(u^{*}\right) \leq 0 \\
d^{L}+\beta_{t} \cdot \sigma \leq d \leq d^{U}-\beta_{t} \cdot \sigma
\end{gathered}
$$

where $G_{p}\left(u^{*}\right)$ is the maximal performance measurement. The purpose of the inner loop is to find $u^{*}$ in $\mathrm{U}$-space such as:

$$
\begin{gathered}
G_{p}\left(u^{*}\right)=\max _{u} G_{U}(u) \\
\text { s.t. }\|u\|=\beta_{t}
\end{gathered}
$$

where $u^{*}$ is the Maximum Performance Target Point (MPTP) that corresponds to the target index $\beta_{t}$.

\subsection{Single-loop methods}

For the so-called single-loop methods, the main point is that the inner loop is replaced by an approximation to avoid the iterative evaluations for reliability analysis in order to accelerate the convergence to the optimum.

\subsubsection{Approximate Moment Approach (AMA)}

AMA is based on statistical moments. The first order Taylor expansion is used to calculate the mean $\mu_{g_{i}}$ and the standard deviation $\sigma_{g_{i}}$ for all constraint functions $g_{i}(d)$ by using the following expressions [9]:

$$
\left\{\begin{array}{c}
\mu_{g_{i}}=g_{i}(d) \\
\sigma_{g_{i}}^{2} \approx\left(\nabla_{g_{i}}^{T}\right)^{2} \cdot \sigma^{2}
\end{array}\right.
$$

where $\sigma$ is the standard deviation of the input variables $X$ and $\nabla$ is the gradient operator. With these expressions, an approximation $g_{i}(d)+\beta_{t} \sigma_{g_{i}}$ is used to replace the original constraints $g_{i}\left(d^{\prime}\right)$ as shown in Figure 3. In this way, the margin of security is shifted in order to keep the reliability as desired. Figure 3 shows the basic idea of AMA approach, $g(d)=0$ is the deterministic limit-state function. As the optimum point $d^{*}$ is on the limit-state curve, the probability of failure is about $50 \%$. The situation which could cause the failure around this point with a given probability is shown as shaded area on the left side. AMA transforms the constraint to find a new optimum point $d^{\prime *}$ on the curve of the new limit-state equation $g(d)+\beta_{t} \sigma_{g}=0$. The situation which could cause the failure around $d^{\prime *}$ is greatly reduced as shown by the shaded area on the right side.

So the problem becomes a deterministic problem:

$$
\begin{gathered}
\min _{d} f(d) \\
\text { s.t. } g_{i}(d)+\beta_{t} \sigma_{g_{i}} \leq 0 \\
d^{L}-\beta_{t} \cdot \sigma \leq d \leq d^{U}+\beta_{t} \cdot \sigma
\end{gathered}
$$
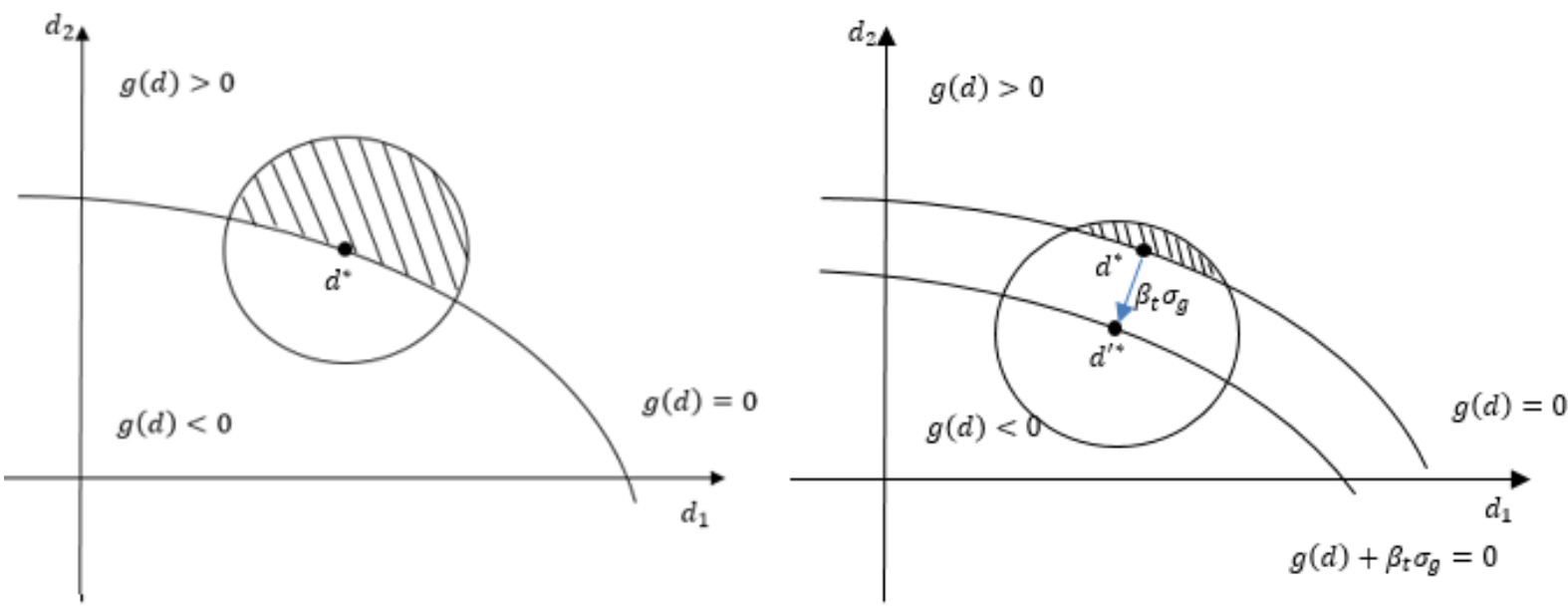

Figure 3. Principle of the AMA approach 
This approach is based on a local linear approximation of the constraint functions at the mean value of the design parameters and as the probabilistic distribution of the performances depends on two moments that are also approximated, the reliability assessment could produce significant numerical error [10].

\subsubsection{Single Loop Approach (SLA)}

SLA uses the same approximation as AMA for calculating the moments and the position of MPPF (marked as $d^{\prime *}$ in Figure 3 and $x_{p}$ in Figure 4). The difference between these two methods is that SLA evaluates the constraints $g$ at the approximate MPPF unlike AMA that uses a first-order approximation of the constraint function around the mean value $d$ directly. The formulation is given as [11]:

$$
\begin{gathered}
\min _{d} f(d) \\
\text { s.t. } g_{i}\left(x_{p_{i}}\right) \leq 0 \\
d^{L}+\beta_{t} \cdot \sigma \leq d \leq d^{U}-\beta_{t} \cdot \sigma
\end{gathered}
$$

with

$$
\begin{gathered}
x_{p_{i}}=d-\beta_{t} \alpha_{i} \circ \sigma \\
\alpha_{i}=\frac{\nabla G_{U_{i}}(d)}{\left\|\nabla G_{U_{i}}(d)\right\|}=\frac{\sigma \circ \nabla g_{i}(d)}{\left\|\sigma \circ \nabla g_{i}(d)\right\|}
\end{gathered}
$$

where $\alpha_{i}$ is the normalized gradient of the $i$ th constraint, $x_{p_{i}}$ is the approximation of the MPPF for the constraint $g_{i}$, and $\circ$ is the Hadamard operator (element-wise) product.

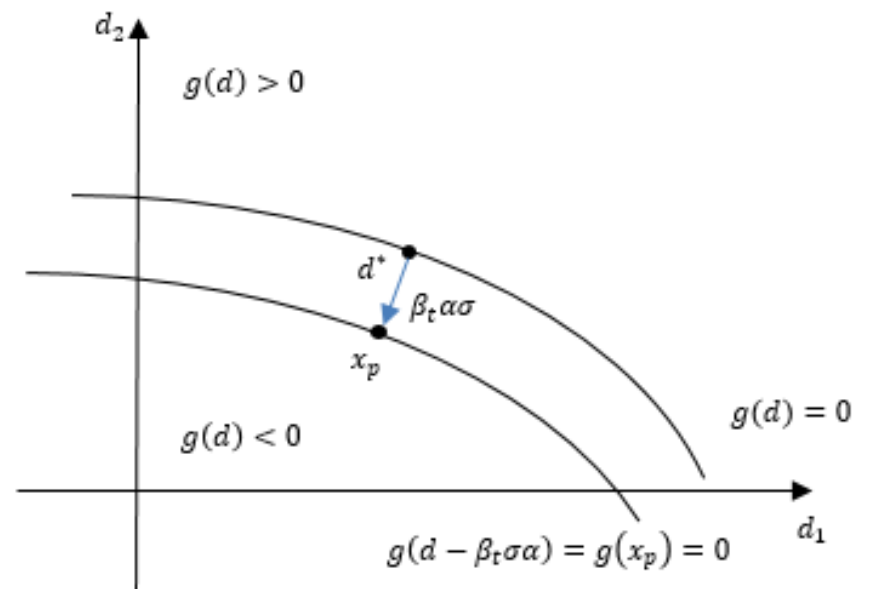

Figure 4. Principle of the SLA approach

The principle of this approach is similar to AMA. As shown in Figure 4, it does not search for the MPPF of each constraint by using an inner loop but approximate its position.

\subsection{Sequential decoupled methods}

Sequential decoupled methods aim to change the initial problem into a series of optimization cycles. The cycles are sequential, each individual optimization is deterministic and uses the optimum given by the former optimization as an initial point. At the first iteration, the algorithm searches a deterministic solution without considering uncertainty and then compute the reliability index of this solution to deduce a shift in order to achieve a given probability of failure. The next iterations refine the shift.

\subsubsection{Sequential Optimization and Reliability Assessment (SORA)}

SORA employs a series of cycles of deterministic optimizations and reliability assessments. In each cycle, optimization and reliability assessment are decoupled from each other, the reliability assessment is only conducted after the deterministic optimization to verify constraint feasibility under uncertainty. The main point of this method is to shift the boundaries of violated constraints to the feasible direction based on the reliability information obtained in the former cycle [12]. The updated point is used in the next cycle of the deterministic optimization. This cycle is repeated until the fulfillment of the convergence criteria.

The process of SORA method is presented as follows. First of all, an initial shift $s^{0}=0$ allows finding the solution $d^{* 0}$ of the deterministic problem. At each iteration, the optimization problem is defined by: 


$$
\begin{gathered}
d^{* k}=\underset{d^{k}}{\operatorname{argmin}} f\left(d^{k}\right) \\
\text { s.t. } g\left(d^{k}-s^{k}\right) \leq 0 \\
d^{L}+\beta_{t} \cdot \sigma \leq d \leq d^{U}-\beta_{t} \cdot \sigma
\end{gathered}
$$

where $k$ indicates the iteration number. The optimal value is set as $d^{* k}$. After each optimization, some of the constraints may become active. For an active constraint, the optimal point $d^{* k}$ is on the limit-state curve. When considering the randomness of $X$, the actual probability of failure is about 0.5 , so a reliability assessment is implemented at the deterministic optimum solution to locate the MPTP $x^{k}$ that corresponds to the desired probability of failure. To ensure the MPTP onto the deterministic boundary, a shifting vector $s^{k+1}$ is derived:

$$
s^{k+1}=d^{* k}-x^{k}
$$

Therefore, when establishing the equivalent deterministic optimization model in the next cycle, the constraints is modified to shift the MPTP at least onto the deterministic boundary.

\subsubsection{Sequential Approximate Programming (SAP)}

SAP is another approach based on Taylor expansion at the first order. The original optimization problem is decomposed into a sequence of sub-optimization problems. Each sub-optimization consists of an approximate objective function subjected to a set of approximate constraint functions [13]. The details are presented as follows:

For considering the PMA formed optimization problems, the expressions are as equation (8), following the idea of SAP, a sequential approximate formulation is constructed as:

$$
\begin{gathered}
\min _{d} f^{k}(d) \\
\text { s.t. } \quad G_{p}\left(d^{k}\right) \leq 0 \\
d^{L}+\beta_{t} \cdot \sigma \leq d \leq d^{U}-\beta_{t} \cdot \sigma
\end{gathered}
$$

The approximate function $G_{p}\left(d^{k}\right)$ is built with a first order Taylor expansion with respect to the design variables at the current point:

$$
G_{p}\left(d^{k}\right) \approx \widehat{G}_{p}\left(d^{k-1}\right)+\left(\nabla_{d} \widehat{G}_{p}\left(d^{k-1}\right)\right)^{T}\left(d^{k}-d^{k-1}\right)
$$

where $\widehat{G}_{p}\left(d^{k-1}\right)=G_{u}\left(u^{k-1}\right)$ is the approximate probabilistic performance measure and $\nabla_{d} \widehat{G}_{p}\left(d^{k-1}\right)$ is its gradient. To avoid another optimization to calculate $u^{k}$, it is updated by the function below:

$$
u^{k}=-\beta_{t} \frac{\nabla_{U} G_{U}\left(u^{k-1}\right)}{\left\|\nabla_{U} G_{U}\left(u^{k-1}\right)\right\|}
$$

where $u^{0}=0$ is usually chosen as the initial estimation.

With the first order approximation of $G_{p}\left(d^{k}\right)$, new formulation is obtained:

$$
\begin{gathered}
\min _{d} f^{k}(d) \\
\text { s.t. } \widehat{G}_{p}\left(d^{k-1}\right)+\left(\nabla_{d} \widehat{G}_{p}\left(d^{k-1}\right)\right)^{T}\left(d-d^{k-1}\right) \leq 0 \\
d^{L}+\beta_{t} \cdot \sigma \leq d \leq d^{U}-\beta_{t} \cdot \sigma
\end{gathered}
$$

This formulation of SAP is similar to the outer loop of PMA. The same principle is used to convert a RIA formulation into a sequential approximate programming [14].

\section{CASE STUDIES}

In this chapter, two examples are tested to compare the performances of all approaches.

\subsection{Numerical example}

To assess the efficiency of these methods, the simple two-variable problem in [15] is analyzed. The optimization problem of this numerical example is: 


$$
\begin{gathered}
\min _{d} f(d)=-\frac{\left(d_{1}+d_{2}-10\right)^{2}}{30}-\frac{\left(d_{1}-d_{2}+10\right)^{2}}{120} \\
\text { s.t. }\left\{\begin{array}{c}
g_{1}=1-\frac{d_{1}^{2} d_{2}}{5} \\
g_{2}=1-\frac{\left(d_{1}+d_{2}-5\right)^{2}}{30}-\frac{\left(d_{1}-d_{2}-12\right)^{2}}{120} \\
g_{3}=1-\frac{80}{\left(d_{1}^{2}+8 d_{2}+5\right)} \\
0 \leq d_{1,2} \leq 10
\end{array}\right.
\end{gathered}
$$

In order to understand the implications of the choice of different formulations and also to quantify the impact of the choice of approaches and algorithms on the accuracy and the number of evaluations, the results of the 7 mentioned methods are given in Table 1. The different optima are compared with each other in Figure 5, where the dotted curves present contours of $f$ and constraint boundaries $g_{i}=0$ are depicted as solid lines. The target probability of failure $P_{t}$ for RBDO is $2.28 \%, P_{f}$ is computed by Monte-Carlo simulation with a sampling of $10^{6}$ realizations. For the simplicity and without loss of generality, all the uncertainties are modeled with the normal law.

Table 1. Results for different optimizations

\begin{tabular}{|c|c|c|c|c|c|c|c|}
\hline Methods & $d_{1}$ & $d_{2}$ & $\mu_{f}$ & $\sigma_{f}$ & $\begin{array}{c}\text { Max of } \\
P_{f}(\%)\end{array}$ & $\begin{array}{c}\text { Convergence } \\
(100 \text { times })\end{array}$ & $\begin{array}{c}\text { Function evaluations } \\
\text { of } f \text { and } g\end{array}$ \\
\hline DDO & 2.4398 & 0.8400 & -2.6266 & 0.2072 & 50 & $20 \%$ & 84 \\
\hline RIA & 2.2513 & 1.9691 & -1.9945 & 0.1792 & 2.28 & $29 \%$ & 4539 \\
\hline PMA & 2.2513 & 1.9691 & -1.9945 & 0.1792 & 2.28 & $82 \%$ & 3183 \\
\hline AMA & 2.3524 & 2.1568 & -1.8712 & 0.1715 & 1.87 & $46 \%$ & 132 \\
\hline SLA & 2.2512 & 1.9677 & -1.9953 & 0.1793 & 2.29 & $32 \%$ & 165 \\
\hline SORA & 2.2513 & 1.9691 & -1.9945 & 0.1792 & 2.28 & $45 \%$ & 531 \\
\hline SAP & 2.2513 & 1.9691 & -1.9945 & 0.1792 & 2.28 & $37 \%$ & 181 \\
\hline
\end{tabular}

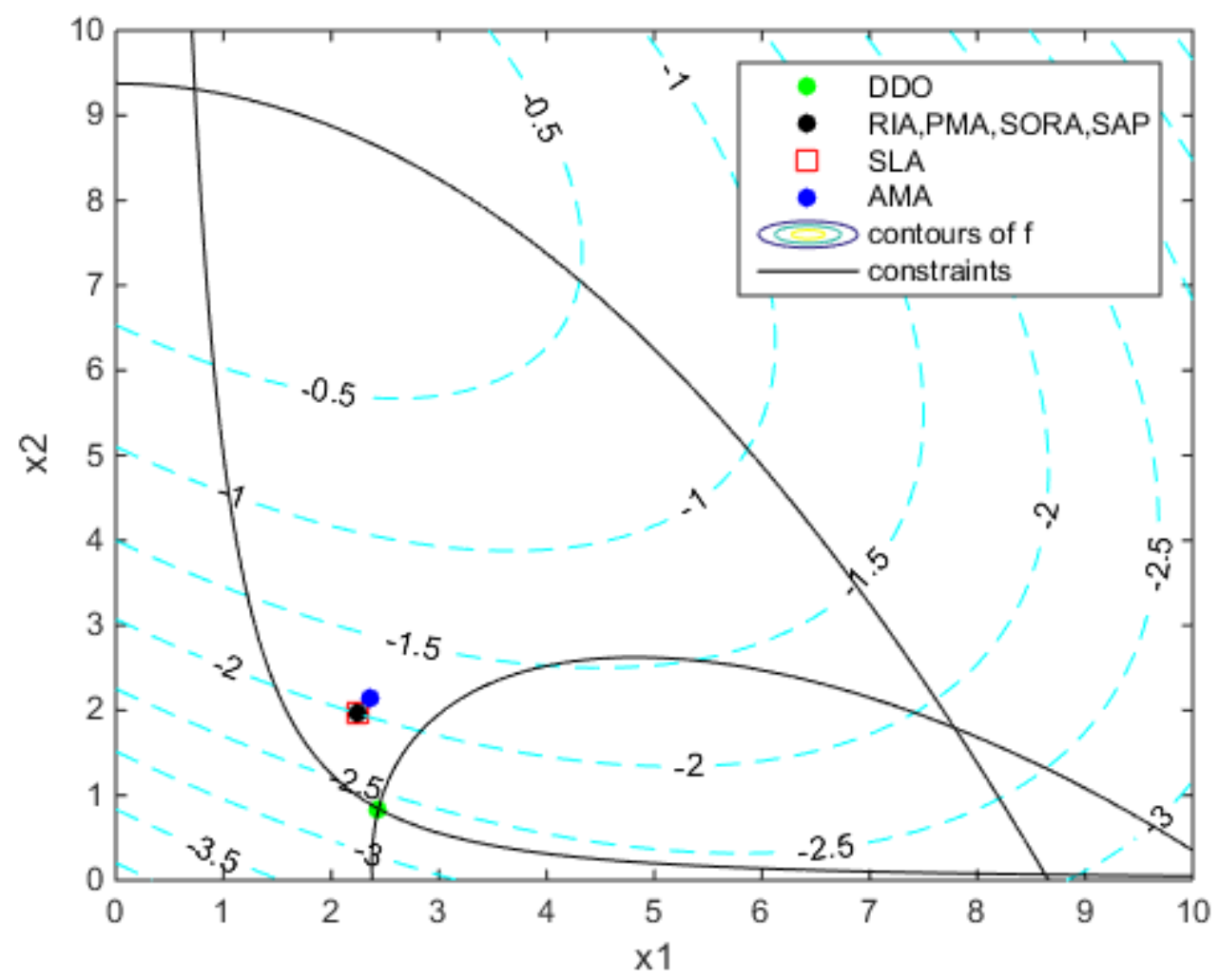

Figure 5. The results of different methods plotted in search space. 
The column named convergence in Table 1 means that we run the algorithms 100 times with different initial points to see how many times it converges to the same optimum. From Table 1 and Figure 5, we can see that most of the methods can find a result that satisfies the constraints. DDO leads to a solution with the lowest objective function mean value, the highest standard-deviation of objective function and a probability of failure around $50 \%$. On the contrary, the probabilities of failure of RBDO methods are close to the target probability except for the single-loop method AMA because of the approximation used to increase the speed of convergence by sacrificing the accuracy. Among RBDO methods, single-loop strategies are fast but inaccurate, double-loop and sequential decoupled methods lead to the same results but sequential decoupled methods are greatly faster.

\subsection{Example of safety transformer optimization}

The example of a safety isolating transformer [16] is also investigated. This is a single-phase transformer with grain-oriented E-I laminations. The primary and secondary windings are both wound around the frame surrounding the central core. The model inputs consist in 7 random design parameters: three parameters $a, b, c$ for the shape of the lamination, one for the frame $d$, two for the section of conductors $S_{1}, S_{2}$ and the last one for the number of primary turns $n_{1}$. These design variables are shown in Figure 6 . The range of input variables are:

$$
\begin{gathered}
3 \mathrm{~mm} \leq a \leq 30 \mathrm{~mm} \\
14 \mathrm{~mm} \leq b \leq 95 \mathrm{~mm} \\
6 \mathrm{~mm} \leq c \leq 40 \mathrm{~mm} \\
10 \mathrm{~mm} \leq d \leq 80 \mathrm{~mm} \\
200 \leq n_{1} \leq 1200 \\
0.15 \mathrm{~mm}^{2} \leq S_{1} \leq 19 \mathrm{~mm}^{2} \\
0.15 \mathrm{~mm}^{2} \leq S_{2} \leq 19 \mathrm{~mm}^{2}
\end{gathered}
$$

There are 8 inequality constraints in this problem. The copper and iron temperatures $T_{\text {cond }}$ and $T_{\text {iron }}$ should be less than given temperatures $120^{\circ} \mathrm{C}$ and $100^{\circ} \mathrm{C}$ respectively. Both the magnetizing current $\frac{I_{10}}{I_{1}}$ and drop voltage $\frac{\Delta V_{2}}{V_{20}}$ should be less than $10 \%$. The filling factors of the primary coil $f_{1}$ and secondary coil $f_{2}$ should be lower than 1 , and the efficiency $\eta$ should be larger than 0.8 . At last, the residue must be less than $10^{-6}$.

The constraint functions are:

$$
g(d)=\left[\begin{array}{c}
T_{\text {cond }}-120 \\
T_{\text {iron }}-100 \\
I_{10} / I_{1}-0.1 \\
\Delta V_{2} / V_{20}-0.1 \\
f_{1}-1 \\
f_{2}-1 \\
0.8-\eta \\
\text { residue }-10^{-6}
\end{array}\right]
$$

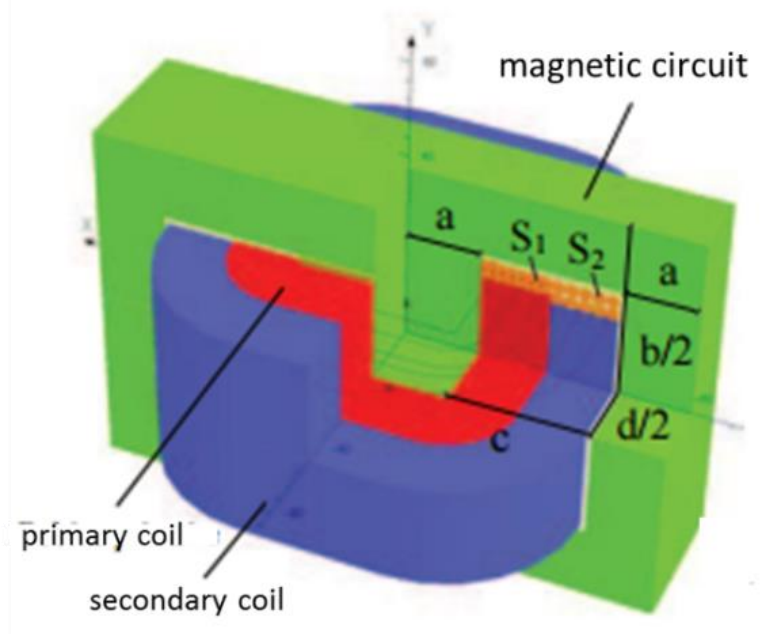

Figure 6. 3D geometric simulation of the transformer 
The aim is to minimize the total mass $M_{\text {tot }}$ with a target probability $P_{t}=0.13 \%$, so that the target reliability index is $\beta_{t}=3$.

Table 2 shows the results of some methods. For each constraint, there are two probabilities of failures, upper ones are calculated by the methods themselves and the below ones are calculated by the Monte-Carlo simulation for comparison. For the reason that the $8^{\text {th }}$ constraint is a residue, we consider it as a deterministic one, so there are only 7 probabilities of constraints presented in the table 2.

Table 2. Results on the safety transformer optimization problem

\begin{tabular}{|c|c|c|c|c|c|}
\hline \multicolumn{2}{|l|}{ Transformer } & DDO & PMA & SLA & SORA \\
\hline \multicolumn{2}{|l|}{$a(m m)$} & 13.004 & 13.078 & 13.045 & 13.078 \\
\hline \multicolumn{2}{|l|}{$b(\mathrm{~mm})$} & 50.1 & 52.274 & 51.613 & 52.278 \\
\hline \multicolumn{2}{|l|}{$c(\mathrm{~mm})$} & 16.537 & 16.758 & 16.837 & 16.757 \\
\hline \multicolumn{2}{|l|}{$d(m m)$} & 43.05 & 42.182 & 42.603 & 42.180 \\
\hline \multicolumn{2}{|l|}{$n_{1}$} & 639.76 & 658.36 & 653.56 & 658.39 \\
\hline \multicolumn{2}{|l|}{$S_{1}\left(m^{2}\right)$} & 0.3238 & 0.3256 & 0.3254 & 0.3256 \\
\hline \multicolumn{2}{|l|}{$S_{2}\left(m^{2}\right)$} & 2.9026 & 2.9296 & 2.9273 & 2.9296 \\
\hline \multicolumn{2}{|l|}{$\mu_{M_{t o t}}$} & 2.3112 & 2.3546 & 2.3534 & 2.3546 \\
\hline \multicolumn{2}{|l|}{$\sigma_{M_{t o t}}$} & 0.0079 & 0.0081 & 0.0081 & 0.0081 \\
\hline \multicolumn{2}{|l|}{ Convergence } & 0.12 & 0.84 & 0.72 & 0.23 \\
\hline \multirow{2}{*}{$P_{f_{1}}\left(T_{\text {cond }}>120^{\circ} \mathrm{C}\right)(\%)$} & by $\mathrm{MC}$ & 0 & 0 & 0 & 0 \\
\hline & by itself & 0 & 0 & 0 & 0 \\
\hline \multirow{2}{*}{$P_{f_{2}}\left(T_{\text {iron }}>100^{\circ} \mathrm{C}\right)(\%)$} & by $\mathrm{MC}$ & 50 & 0.13 & 0.13 & 0.13 \\
\hline & by itself & 50.658 & 0.1337 & 0.3754 & 0.1389 \\
\hline \multirow{2}{*}{$P_{f_{3}}\left(\Delta V_{2} / V_{20}>0.1\right)(\%)$} & by $\mathrm{MC}$ & 0 & 0 & 0 & 0 \\
\hline & by itself & 0 & 0 & 0 & 0 \\
\hline \multirow{2}{*}{$P_{f_{4}}\left(I_{10} / I_{1}>0.1\right)(\%)$} & by $\mathrm{MC}$ & 50 & 0.13 & 0.13 & 0.13 \\
\hline & by itself & 50.203 & 0.1356 & 0.1357 & 0.1370 \\
\hline \multirow{2}{*}{$P_{f_{5}}\left(f_{1}>1\right)(\%)$} & by $\mathrm{MC}$ & 50 & 0.13 & 0.13 & 0.13 \\
\hline & by itself & 49.987 & 0.1323 & 0.1362 & 0.1388 \\
\hline \multirow{2}{*}{$P_{f_{6}}\left(f_{2}>1\right)(\%)$} & by $\mathrm{MC}$ & 50 & 0.13 & 0.13 & 0.13 \\
\hline & by itself & 50.021 & 0.1415 & 0.1368 & 0.1382 \\
\hline \multirow[b]{2}{*}{$P_{f_{7}}(0.8-\eta)(\%)$} & by $\mathrm{MC}$ & 50 & 0.13 & 0.13 & 0.13 \\
\hline & by itself & 49.757 & 0.1324 & 0.1376 & 0.1390 \\
\hline \multicolumn{2}{|c|}{ Model evaluation } & 242 & 30607 & 6048 & 2171 \\
\hline
\end{tabular}

Note that PMA can find an optimum that satisfies all the constraints even if the number of evaluations of the model is high. For SLA, it has a smaller number of evaluations but there is one constraint violated. The reason is that SLA sacrifices the accuracy in order to reduce the number of evaluations, leading to a coarse computation of the probability of failure. The convergence rate of SORA is not as good as the other two but it has the smallest number of evaluations among them. It can be seen that this number is nearly 15 times less than PMA and 3 times less than SLA. Unfortunately, the rate of convergence for SORA is 4 times lower. This means that a multi-start process is required and the number of evaluations will increase consequently. We obtain almost the same conclusions as with the numerical example: the single-loop method SLA is the most inaccurate method and doubleloop method PMA has the highest convergence rate. For this more complicated example, the number of evaluations of sequential decoupled method SORA is even smaller than of SLA, so that the more complex the model is, the more effective SORA may be. Other methods fail to find a solution, probably because this problem is hardconstrained and the solution of DDO is on the limit-state of four constraints. So, it also indicates that not all the aforementioned approaches can handle complicated models.

\section{CONCLUSION}

RBDO methods change the initial constraints into probabilistic ones and use different approaches to approximate the limit state function or the most probable point of failure to calculate the probability of failure or the reliability index.

The mathematical example shows that the 6 RBDO approaches have almost the same results except AMA that is less accurate. The optimization of the safety transformer highlights that not all the methods can converge to 
the global solution. PMA, SLA, and SORA appear to be more stable. Considering both numerical examples, SORA is the most effective method among all RBDO approaches.

\section{REFERENCES}

[1] Xiao, S., Li, Y., Rotaru, M., Sykulski, J. K. (2014), "Considerations of uncertainty in robust optimisation of electromagnetic devices", International Journal of Applied Electromagnetics and Mechanics, Vol. 46 No. 2, pp. 427-436.

[2] Kim, D. W., Kang, B., Choi, K. K., et al. (2016), "A Comparative Study on Probabilistic Optimization Methods for Electromagnetic Design", IEEE Transactions on Magnetics, Vol. 52 No. 3, pp. 1-4.

[3] Ren, Z., Zhang, D., and Koh, C. S. (2016). "Investigation of reliability analysis algorithms for effective reliability-based optimal design of electromagnetic devices", IET Science, Measurement \& Technology, Vol. 10 No. 1, pp. 44-49.

[4] Liu, P.L., Der Kiureghian, A. (1991), "Optimization algorithms for structural reliability", Structural safety, Vol. 9 No. 3, 1991, pp. 161177.

[5] Hasofer, A.M. and Lind, N.C. (1974), "Exact and invariant second-moment code format. American Society of Civil Engineers", Engineering Mechanics Division, Vol. 100, pp. 111-121.

[6] Tu, J., Choi, K.K., and Park, Y.H. (1999), "A new study on reliability-based design optimization", Journal of mechanical design, Vol. 121 No. 4, pp. 557-564.

[7] Wu, Y.T., Millwater, H.R., Cruse, T.A. (1990), "Advanced probabilistic structural analysis method for implicit performance functions", AIAA journal, Vol. 28 No. 9, pp. 1663-1669.

[8] Youn, B.D., Choi, K.K. and Park, Y.H. (2003), "Hybrid analysis method for reliability-based design optimization", Journal of Mechanical Design, Vol. 125 No.2, pp. 221-232.

[9] Putko, M.M, Taylor, A.C., Newman, P.A., et al. (2002), "Approach for input uncertainty propagation and robust design in CFD using sensitivity derivatives", Journal of Fluids Engineering, Vol. 124 No. 1, pp. 60-69.

[10] Youn, B.D., and Choi, K.K. (2004), "Selecting probabilistic approaches for reliability-based design optimization", AIAA journal, Vol. 42 No. 1, pp. 124-131.

[11] Liang, J., Mourelatos, Z.P., and Tu, J (2004), "A single-loop method for reliability-based design optimization", in ASME 2004 International Design Engineering Technical Conferences and Computers and Information in Engineering Conference, 2004, Salt Lake City, pp. 419-430.

[12] Du, X., and Chen, W. (2004), "Sequential optimization and reliability assessment method for efficient probabilistic design)", Journal of Mechanical Design, Vol. 126 No. 2, pp. 225-233.

[13] Yi, P., Cheng, G., and Jiang, L. (2008), "A sequential approximate programming strategy for performance-measure-based probabilistic structural design optimization", Structural Safety, Vol. 30 No. 2, pp. 91-109.

[14] Cheng, G., Xu, L., and Jiang, L. (2006), "A sequential approximate programming strategy for reliability-based structural optimization", Computers \& structures, Vol. 84 No.21, pp. 1353-1367.

[15] Kim, D.W., Choi, N.S., Choi, K.K. et al. (2015), "A Single-Loop Strategy for Efficient Reliability-Based Electromagnetic Design Optimization", IEEE Transactions on Magnetics, Vol. 51 No. 3, pp. 1-4.

[16] Tran, T.V., Brisset, S. and Brochet, P. (2007), "A Benchmark for Multi-Objective, Multi-Level and Combinatorial Optimizations of a Safety Isolating Transformer", in 16th International Conference on Computation of Electromagnetic Fields, 2007, Aachen, pp. 167-168.

[17] Lopez, R.H. and Beck, A.T. (2012), "Reliability-based design optimization strategies based on FORM: a review", Journal of the Brazilian Society of Mechanical Sciences and Engineering, Vol. 34 No. 4, pp. 506-514. 